\title{
TOWARDS THE CONSTRUCTION OF THE MULTIMEDIA MEDIATION MECHANISM
}

\author{
Masao Sakauchi
}

\author{
Institute of Industrial Science, University of Tokyo \\ 7-22-1 Roppongi, Minato-ku, Tokyo 106-8558, Japan \\ sakauchi@sak.iis.u-tokyo.ac.jp
}

\begin{abstract}
Multimedia Information Environments based on video are now growing rapidly. Especially three types of environments, i.e. "Stream type Multimedia Environment" by digital TV broadcasting, "Real-world type Multimedia Environment" monitoring roads and towns, and "Network type Multimedia Environment" on the WWW are especially promising targets. In this paper, a new framework for developing applications and services from these three Multimedia Environments named by "the Multimedia Mediation Mechanism", is proposed and discussed. In the Stream type Multimedia Mediation System, basic functions including video stream description, data retrieval, data integration, event discovery and data creation are designed. New interactive video services, personal media services are developed on the network as application. In the Real-world type Multimedia Mediation System, basic functions including construction of mediation map, locating functions, object recognition and event discovery are designed. Several applications for ITS (Intelligent Transport System) etc. are also developed. In the Network type Multimedia Mediation System, various advanced search engines are developed.
\end{abstract}

\subsection{INTRODUCTION}

Rapid expansion of multimedia information space based on video or image data is being realized by means of various distribution tools. Three types of multimedia information spaces (or environments), i.e. "in the digital broadcasting stream", "in the real-world" and "on the Internet" are especially promising. On the other hand, from social and economic viewpoints, importance of infor- 
mation processing techniques which can create real value for human activity or life should be surely recognized.

Considering these two backgrounds, we are now developing a new multimedia database system, named the Multimedia Mediation Mechanism (or System) of application-oriented middleware for realization of functions, services demanded by human and society.

In this paper, the framework of the Multimedia Mediation System, the basic functions for realizing three types of concrete Multimedia Mediation Systems of Stream type Multimedia System, Real-world type Multimedia System and Network type Multimedia System will be discussed with several embodiment, mainly based on our research project (http://shinpro.sak.iis.u-tokyo.ac.jp/indexe.html).

\subsection{THREE TYPES OF “MULTIMEDIA ENVIRONMENT" AND THE MULTIMEDIA MEDIATION SYSTEM}

Fig. 2.1 shows the framework of the Multimedia Mediation System (MMS). The MMS consists of three types of individual mediation system, i.e. the Stream type MM System, the Real-world type MM System and the Network type MM System, corresponding to Stream type, Real-world type and Network type MM Environment, respectively.

\section{〈Stream type MMS 〉}

Needless to say, one of the typical leaders of multimedia information (contents) providers is broadcasting. Commercial-based satellite digital broadcasting with over hundreds of channels have already started in USA, Japan, Europe and Asia. Another digital broadcasting in the form of CATV, ordinary surface wave TV, or Fibernet communication also have already been or will be planed soon. In such situations, where we'll be able to enjoy hundreds or thousands of broadcasting channels, much more user-oriented and intelligent access to the tremendous amount of "contents stream" will be required as shown in the top-left part of Fig. 2.1.

In this Stream type MMS, the mediator functions would be most important for user, which help them to pick up useful and required data from the stream and to make their own customized contents for new service and business.

\section{〈Real-world type MMS 〉}

We Japanese, suffered severe earthquake damage at Kobe in January, 1995. This tragedy taught us the importance of realtime acquisition of our city information for disaster mitigation. Multimedia communication technology enables us to establish a new type of database which collects and analyzes realtime situations (video information) to tell us "What's going on in the city" on realworld. Let's call this type as "Real-world type MM Environment" as shown in the top-middle part of Fig. 2.1.

Though this type of systems generally have not been considered as multimedia system, they have promising possibility to realize new services and busi- 

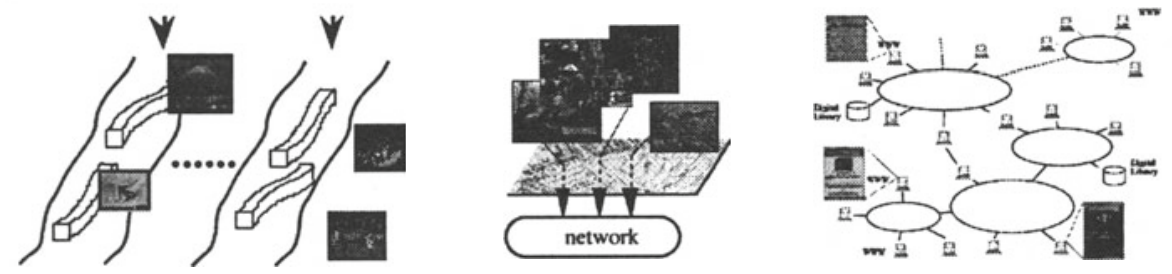

Stream type MM Environment

Real-world type MM Environment

Network type MM Environment

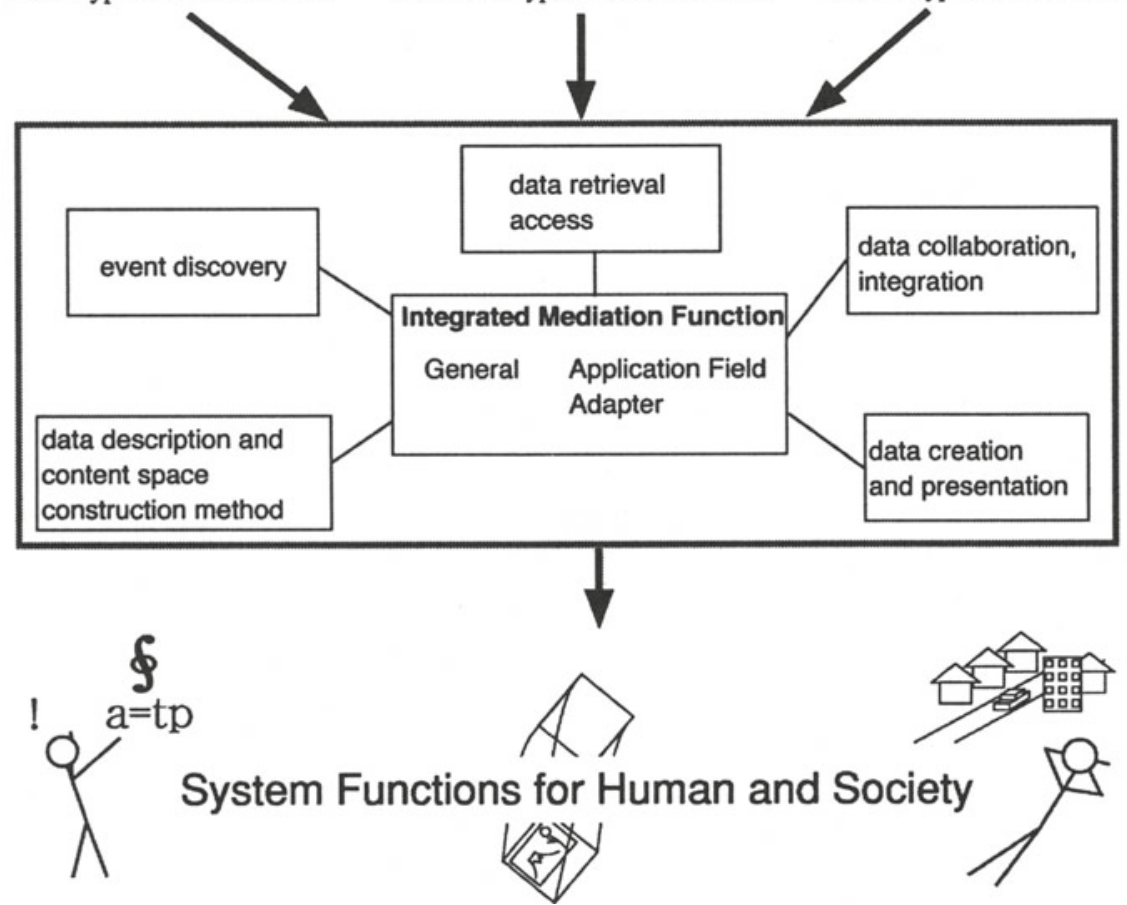

Figure 2.1: Framework of Multimedia Mediation System

ness with real time integration of real-world situations and existing another databases. Expecting fields in this category include disaster mitigation, intelligent transport systems (ITS), advanced environment management, advanced utilization of high resolution satellite images etc.

\section{〈Network type MMS 〉}

The "Internet" data services including the WWW are now becoming more and more popular. Tremendous amount of services provide multimedia information even in the form of video and images. Hereafter, this means that most 
databases will be scattered where contents are generated and such situations will grow uncontrollably. Such dispersed databases should be called here the "Network type MM Environment" as one of our promising targets as shown in the top-right part of Fig. 2.1.

There are many "what to do" for this environment. We believe, however, mediator functions, which inform users what kinds of data exist in the network, or where is their useful or desired information, and help to collect and relate them to the user's applications, are the most important. Such "contexing" of the network multimedia data should be the main function in the database. Their typical and first-step trials are the "Yahoo" directory service or another search engines for text data. Various trials and functions, however, will be required for more general multimedia data in the network.

Basic functions in order to create various services and applications from these three environments are the core part of the MMS as shown in the lower part of Fig. 2.1. In the followings, more concrete discussion for each individual MM systems, will be given based on our research project.

\subsection{STREAM TYPE MULTIMEDIA MEDIATION SYSTEM}

More detailed basic mediation functions for the Stream type MMS are shown in Fig. 2.2. In this case, target multimedia data include video stream in digital broadcasting, video contents etc. New interactive video services and personal media services are examples of target applications.

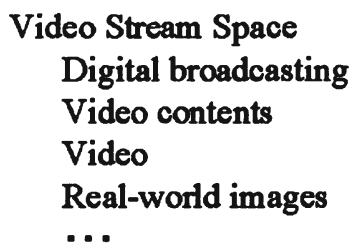

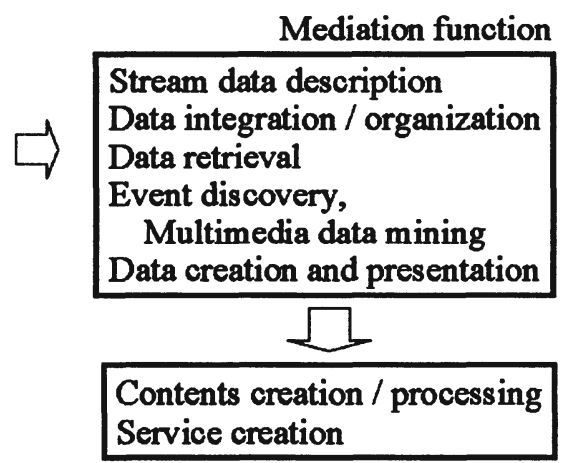

New interactive video service Personal media service

Figure 2.2: Stream type Multimedia Mediation System

Detailed mediation functions include stream data description, data integration / organization, data retrieval, event discovery / multimedia data mining, 
data creation and presentation for applications. Concrete examples in our project for Stream type MM functions are listed below.

\section{Stream data description functions}

- Interactive description [6]

- Interactive object description on the network

- Automatic description [7][8][9][10]

- Object description using synchronized video, audio and documents

- Advanced video analysis using natural language processing

- Face identification system using video and transcripts

\section{Data retrieval, Presentation functions}

- Live hypermedia system [11][12]

- Automated picking up of video sequence by Scene Description Language

- Music stream data retrieval

- Music retrieval using musical interval, melody and lyrics

- Data creation / Presentation [13][14]

- Video editing based on script processing

Because we have no room to describe about those researches in detail, please access to references or (http://shinpro.sak.iis.u-tokyo.ac.jp/index-e.html). Only several examples are overviewed here.

Fig. 2.3 shows the framework adopted for description of video data, where description labels for both video frame (scene) and objects in the frame. Those descriptions are written by object-oriented video scene description language SVSDL. Various operations for management of these description have been developed by Java environment.

Fig. 2.4 illustrates an example of these basic operations. Contents of video description in this example are generated automatically, by our video understanding method using logical synchronization among video, sounds and document based on devised DP matching technique.

Fig. 2.5 shows an example of "edit operation" of video streams. A user can easily realize his/her own video authoring just by using much easier text processing (editing), because video streams have tight timing synchronization with scenario documents by SVSDL description. 


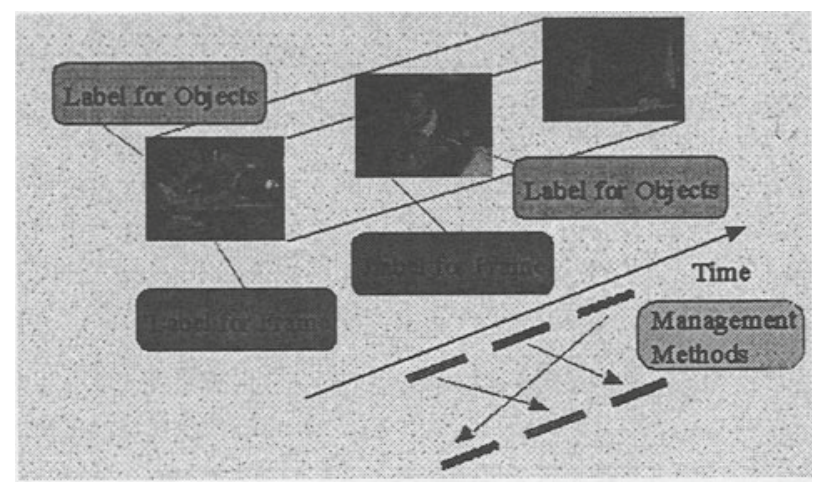

Figure 2.3: Framework of Video Data Description

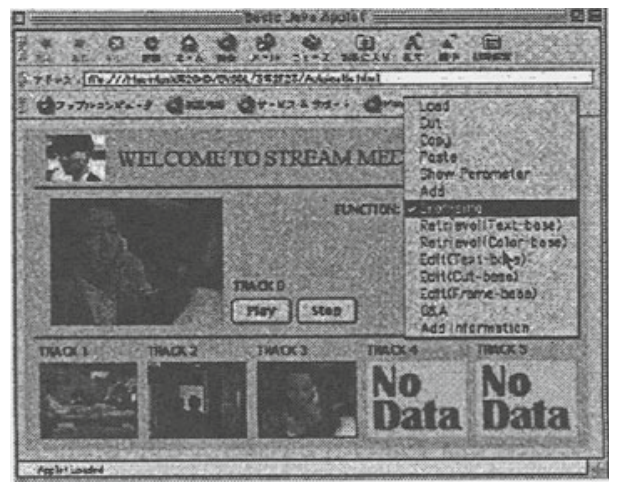

Figure 2.4: Prototype System 


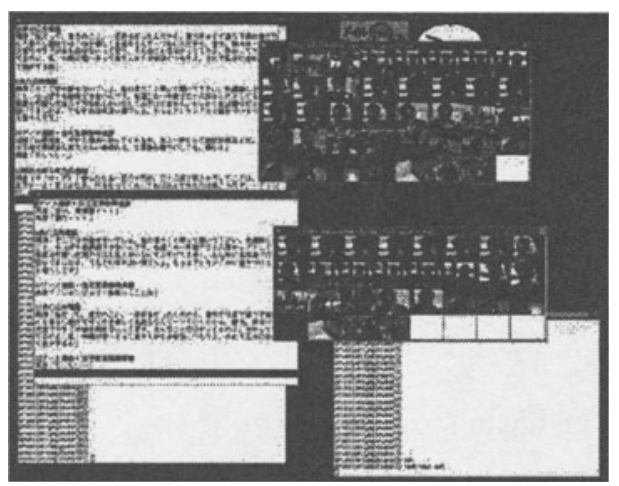

Figure 2.5: Editing by Script

\subsection{REAL-WORLD TYPE MULTIMEDIA MEDIATION SYSTEM}

Fig. 2.6 shows more detailed basic mediation function for the Real-world type MMS. In this case, the target multimedia data include various data from robot cameras, mobile units or network sites, reflecting realtime situations in the rea!world, such as town scenes or traffic on the roads. Intelligent transport system (ITS), various applications for town life, social security system are examples of target applications.

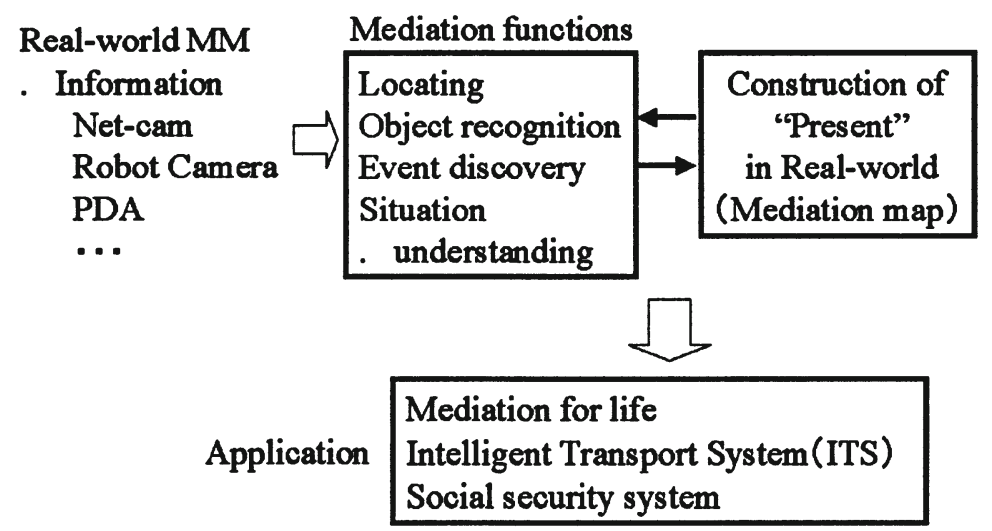

Figure 2.6: Real-world type Multimedia Mediation System

Detailed mediation functions include contraction of present situations in the real-world, locating function for objects in images or video, object recognition, 
event discovery from real world images or video stream. Concrete examples in our project for Real-world type MM functions are listed below.

- Object description, mediation map construction function [15]

- Object description using digital city map and video for urban scene

- Object description function [16]

- Extraction of 3D information from video of traffic

- Data creation, mediation map function [17]

- Realization of AR by acquiring a radiance distribution

- Others

- Mediation function for Real-world contents (Map, Net-cam, High resolution satellite image etc.)

Only two of those are overviewed below. Fig. 2.7 shows one method of object description (or recognition) of urban scenes in distant views. Considering urban scenes in distant views, the buildings which are very high or have special shapes are very prominent. We propose an approach for understanding urban scenes in distant views first by recognizing key buildings appearing as silhouette using a model-based object recognition scheme. A city map-database with 3D descriptions of the rooftops of the buildings is used to build a world model. The feature correspondences between the images and the model are established using a dynamic programming technique. Rough viewing parameters for location and orientation can be obtained from the sensors. Although these parameters are not precise, the information about which buildings appear as silhouettes can be obtained. Based on this information, a model consisting of building's rooftop line segments is constructed for recognition. The feature correspondences between the images and the model are established using a dynamic programming technique. The correspondence hypotheses are then verified to ensure that the correspondences are reliable and accurate. Incorrect feature correspondences caused by sensor uncertainty and image clutter are modified, based on a similarity evaluation method. Using this method, the buildings appearing as silhouette in distant views can be identified as shown in Fig. 2.7.

Fig. 2.8 shows another method of recognizing urban scenes in close-range views. In the case of close-range images, only part of a building's surface can be seen clearly instead of building's shape. Therefore, we prefer to use appearance matching rather than shape matching used in recognizing buildings in distant view. For the recognition of a single building, we use an eigen window method which is an effective appearance-based method. The space being modeled by the eigen windows is intensive patterns in the image. The reliability of the eigen window method is also discussed under the transformations 


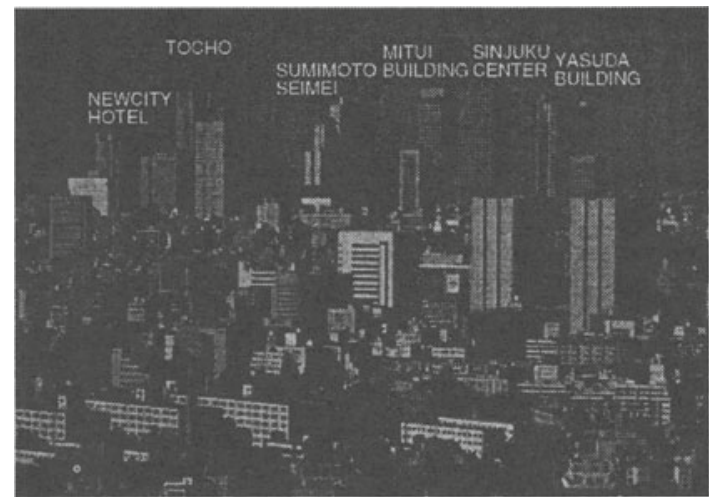

Figure 2.7: Recognizing Buildings in Distant View

of translation, rotation, scale, and viewing angle. When a number of buildings are present in an image, like scenes we see while walking along a road, we segment the image into building regions based on edge distributions. A building in a separated region can be recognized by the same method as recognizing a single building. However, considering that there are many buildings in an urban scene, the recognizing process will be time-consuming, and it is possible that some buildings might be in similar appearance. Fortunately, buildings with similar appearances are often on different streets. A digital map provides spatial relations of buildings, for example, along the Aoyama St. in Tokyo there is Honda building and beside Aoyama Twin Tower. We build a building image database having the same relations as in the map to support recognition. Images of these buildings are shown on the left of Fig. 2.8. We use the features of extracted building regions such as color to represent the context of a scene, and use this description to retrieve candidate roads from the building image database. The retrieval result is shown on the right of Fig. 2.8. We build eigen spaces for the buildings along each road. A building in a separated region can be recognized by being projected into eigen spaces of the candidate roads. Therefore the retrieval results can be verified.

\subsection{NETWORK TYPE MULTIMEDIA MEDIATION SYSTEM}

Fig. 2.9 shows more detailed basic mediation functions for the Network type MMS. In this case, mediation functions for realizing advanced search engine or mediation for solution include Event discovery (data mining),data retrieval, data collaboration or interface for mediation.

Concrete examples in our project for Network type MM functions are listed below.

- Data retrieval function [18][19] 

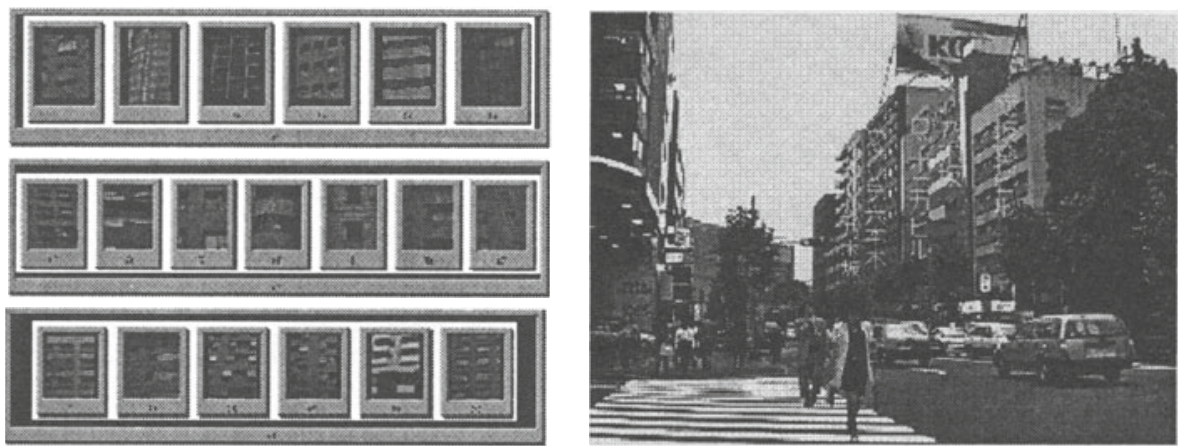

Figure 2.8: Recognizing Buildings in Close Range View

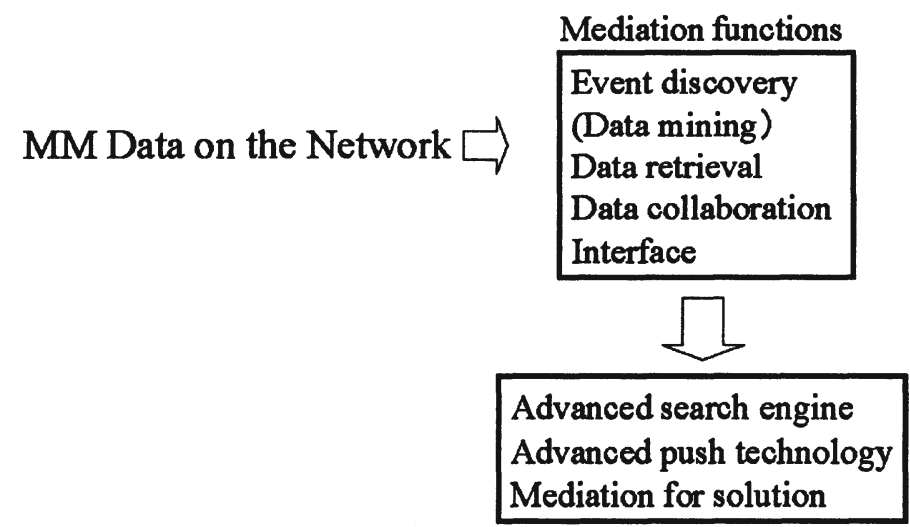

Figure 2.9: Network type Multimedia Mediation System 
- Advanced search engine

- Data Collaboration function [20]

- Data organization using concept information system

- Collaboration based on visualization of information mediation

- Others [21]

- High speed data mining mechanism etc.

Fig. 2.10 shows operation of GIRLS (Global Image Retrieval and Linking System) as one of these examples. GIRLS for the image data in the WWW has been developed as the embodiment of such database systems. In GIRLS, a search robot named BOYS automatically gathers typical images and layout information and URL from the WWW to construct the database for retrieval and linking based on the image contents. The system provides users with open functions including retrieval of images or logo's in the WWW data space, linkage to the original homepages with images imagined by users, feedback to the retrieval performance. In the example for the GIRLS operation in Fig. 2.10, similarity retrieval results to the given layout homepage are shown.
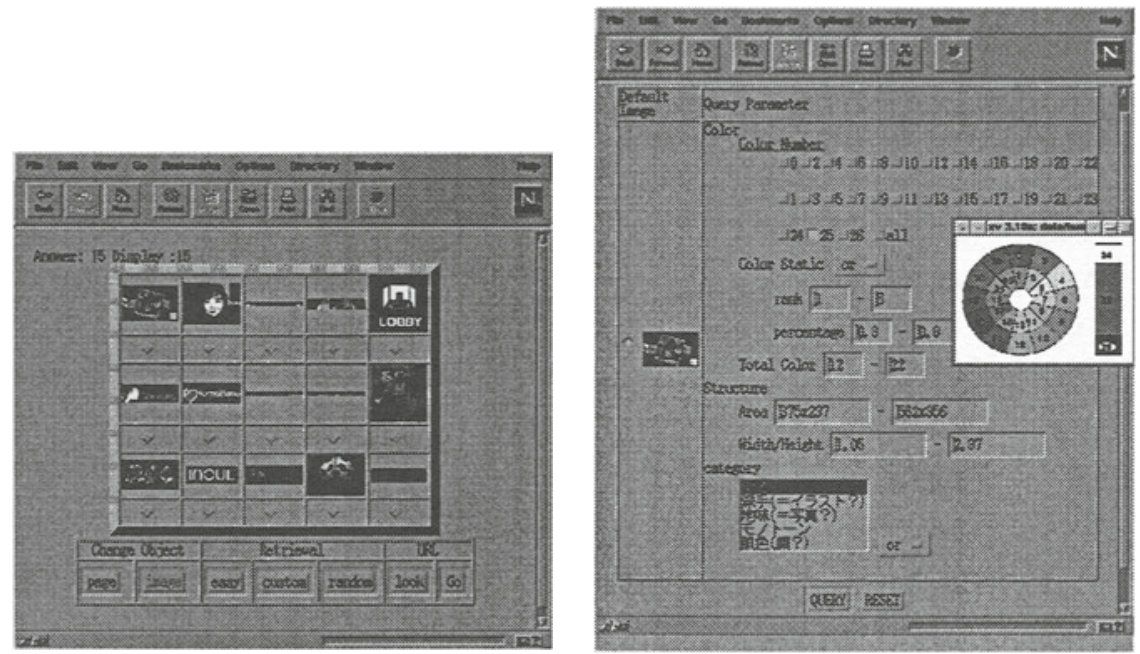

Figure 2.10: Image Search Engine GIRLS (Global Image Retrieval and Linking System) 


\subsection{CONCLUSIONS}

In this paper, the Multimedia Mediation System has been proposed and discussed in order to provide various applications and services. Three types of individual systems include promising field of digital broadcasting applications, ITS applications and network search engines.

Our project is now growing toward realization of useful middleware for multimedia data spaces.

\section{Acknowledgments}

This work is supported as the Grant-in-Aid for Creative Basic Researh \#09NP1401: "Research on Multimedia Mediation Mechanism for Realization of Human-oriented Information Environments" by the Ministry of Education, Science, Sports and Culture, Government of Japan.

\section{References}

[1] M.Sakauchi, "Video Information Media from the Viewpoint of Computer - Proposal of Multimedia Date Mediator," The Journal of the Institute of Image Information and Television Engineers, vol.51, no.1, 1997

[2] M.Sakauchi, T.Satou and Y.Yaginuma, "Multimedia database systems for the contents mediator," The Transaction of the Institute of Electronics, Information and Communication Engineers, vol.E79-D, no.6, pp.641-646, 1996

[3] Y.Yaginuma, T.Yatabe, T.Satou, J.Tatemura and M.Sakauchi, "Four Promising Multimedia Databases and Their Embodiments," Multimedia Tools and Applications, Kluwer Academic Publishers, 5, pp.65-77, 1997

[4] M.Sakauchi, "Image retrieval and image understanding," IEEE Multi-media Computing Magazine, vol.1, no.1, pp.79-81, 1994

[5] M.Sakauchi, Y.Ohsawa and T.Sagara, "Construction and application of multimedia geographical database," Journal of Institute of Geographical Information Systems of Japan, vol.3, no.2, pp.53-58, 1995

[6] T.Yatabe, T.Ohba and M.Sakauchi, "A Proposal of Structured Video Database Using Multiple Users' Description on the Network," Technical Report of the Institute of Electronics, Information and Communication Engineers, PRMU97-64, pp.63-68, 1997

[7] Y.Yaginuma and M.Sakauchi, "Construction of Intelligent TV Drama Database based on the Synchronization between Image, Sound and Script," Proc. of Pacific Workshop on Distributed Multimedia Systems, pp.125-132, 1996

[8] Y.Yaginuma and M.Sakauchi, "A proposal of the synchronization method between drama image, sound and scenario document using DP matching," The Transaction of the Institute of Electronics, Information and Communication Engineers, vol.J79-D-II, no.5, pp.747-755, 1996 
[9] N.Izumi, Y.Yaginuma, H.Nakagawa and M.Sakauchi, "Construction of Existence/Action Map Based on the Script Analysis," The Journal of The Institute of Electronics, Information and Communication Engineers D-II, vol.J79-D-II, no.11, pp.1993-1996, 1996

[10] S.Satoh and T.Kanade, "Name-It: Association of face and name in video," Proc. of Computer Vision and Pattern Recognition, pp.368-373, 1997

[11] T.Satou and M.Sakauchi, "Data acquisition in live hyper media," Proc. of IEEE International Conference on Multimedia Computing and Systems '95, pp.175-181, 1995

[12] T.Satou and M.Sakauchi, "Video information acquisition on live hypermedia," The Journal of The Institute of Electronics, Information and Communication Engineers, vol.J79-D-II, no.4, pp.559-567, 1996

[13] Y.Yaginuma and M.Sakauchi, "Content-based Drama Editing based on Inter-media Synchronization," Proc. of IEEE International Conference on Multimedia Computing and Systems '96, pp.322-329, 1996

[14] Y.Yaginuma and M.Sakauchi, "A proposal of a video editing method using synchronized scenario document," The Transaction of the Institute of Electronics, Information and Communication Engineers, vol.J79-D-II, no.4, pp.547-558, 1996

[15] P.Liu, W.Wu, K.Ikeuchi and M.Sakauchi, "Recognition of Urban Scene Using Silhouette of Buildings and City Map Database," Proc. of the 3rd Asian Conference on Computer Vision, 1998

[16] C.X.Li, P.T.Wang, H.T.Zen and M.Sakauchi, "Creation of PlaneSpatiotemporal-Image Using a Selected Slit," Proc.of IAPR Workshop on Machine Vision Applications '96, 1996

[17] I.Sato, Y.Sato and K.Ikeuchi, "Seamless Integration of Computer Generated Objects into a Real Scene Based on a Real Illumination Distribution," The Transaction of the Institute of Electronics, Information and Communication Engineers, vol.J81-D-II, no.5, pp.861-871, 1998

[18] T.Yatabe, H.Takaha, T.Satoh and M.Sakauchi, "Open Image Search Engine System GIRLS using image infomation," Proc. of Advanced Database Symposium, pp.139-145, 1996

[19] S.Sugawara, K.Yamamoto and Y.Sakai, "A Study on Image Searching Method in Super Distributed Database," Proc. of IEEE GLOBECOM '97, vol.2, pp.736, 1997

[20] Y.Takama and M.Ishizuka, "Application of Fish Eye Vector Based on Concept Structure for Information Organization Supporting Tool," Technical Report of Japan Society for Artificial Intelligence, SIG-FAI-9702-17, pp.97102, 1997

[21] T.Shintani and M.Kitsuregawa, "Parallel Mining Algorithms for Generalized Association Rules with Classification Hierarchy," Proc. of ACM SIGMOD International Conference on Management of Data, 1998 